\title{
Lazarus syndrome in nonsmall cell lung cancer patients with poor performance status and major leukocytosis following nivolumab treatment
}

To the Editor:

Immune checkpoint inhibitors (ICI) have been shown, in the second-line setting, to increase survival in unselected patients with nonsmall cell lung cancer (NSCLC) and good Eastern Cooperative Oncology Group (ECOG) performance status (PS 0-1), compared with standard chemotherapy [1-3]. Nonetheless, the efficacy of these agents when administered to patients with poor PS remains largely unexplored. We report on two patients with advanced NSCLC, both with major features of systemic inflammation and poor general condition (PS 3-4), who manifested a dramatic response to nivolumab.

Patient 1 was a 47-year-old woman (figure 1a), who was a heavy smoker and experiencing thoracic pain. Computed tomography (CT) demonstrated an $80 \mathrm{~mm}$ mass visible in the right lung, as well as a contralateral nodule (cT4N0M1a; Union for International Cancer Control (UICC), version 8). CT-guided biopsies revealed an NSCLC-NOS (not otherwise specified), which was transcription termination factor-1 (TTF-1)- and p40-negative, with marked neutrophil infiltration. Extensive next-generation sequencing analyses failed to detect any additive oncogenic mutation. In March 2016, the patient's PS was 0, and she received two cycles of platinum-pemetrexed doublet as first-line therapy. Although she did not display any specific toxicity, she experienced a dramatic progression of her thoracic disease. Her general condition worsened to PS 4, and she experienced intense parietal chest pain and consciousness disturbances, yet without any brain abnormalities on magnetic resonance imaging suggestive of brain or leptomeningeal metastases. Cytological analysis of cerebrospinal fluid proved negative for tumour cells. A fever reaching a plateau of $38.5^{\circ} \mathrm{C}$ occurred, associated with increased peripheral blood leukocyte levels (up to 24000 per $\mathrm{mL}$, with $80 \%$ neutrophils), without any evidence of infection being revealed by multiple blood and sputum cultures, in addition to serological tests. Blood C-reactive protein (CRP) and lactate dehydrogenase (LDH) levels were respectively increased up to 30 and 2.5 times the normal values, reflecting the patient's inflammatory status. There were no plausible explanations for this general and neurological deterioration associated with inflammatory signs evoking a paraneoplastic syndrome.

Owing to the strong ( $>90 \%)$ PD-L1 expression on the tumour membrane shown by immunohistochemistry using E1L3N monoclonal antibody (Cell Signaling Technology, Leiden, The Netherlands) with a Leica Bond III autostainer (Leica Biosystems, Nanterre, France), along with the strong tumour-infiltrating immune PD-L1 staining of the CT-guided diagnostic biopsies sampled prior to first-line chemotherapy, nivolumab infusion was administrated despite the patient's poor PS. The patient was then transferred to a palliative care unit. Her general condition improved spectacularly within 10 days, allowing her to be discharged from hospital on Day 15. She experienced complete pain relief, enabling all analgesic agents to be discontinued. With nivolumab infusions continued every 15 days, CT scanning revealed major partial response after four infusions, and the leukocytosis decreased quickly. Ten months after the first nivolumab infusion, while still receiving nivolumab every other week, the patient returned to work. At Month 9, a potential treatment-related pneumonitis was diagnosed by CT scan, based on the presence of diffuse ground-glass opacities. Of note is that the patient did not experience any dyspnoea, cough or hypoxia. Although no infectious agents were detected in broncho-alveolar lavage fluid, the diagnosis of CD8+ T-lymphocyte

@ERSpublications

Nivolumab appears efficacious in highly PD-L1-expressing NSCLC with poor PS, resulting in Lazarus syndrome http://ow.ly/X92e30coTsB

Cite this article as: Pluvy J, Brosseau S, Naltet C, et al. Lazarus syndrome in nonsmall cell lung cancer patients with poor performance status and major leukocytosis following nivolumab treatment. Eur Respir J 2017; 50: 1700310 [https://doi.org/10.1183/13993003.00310-2017]. 
a)

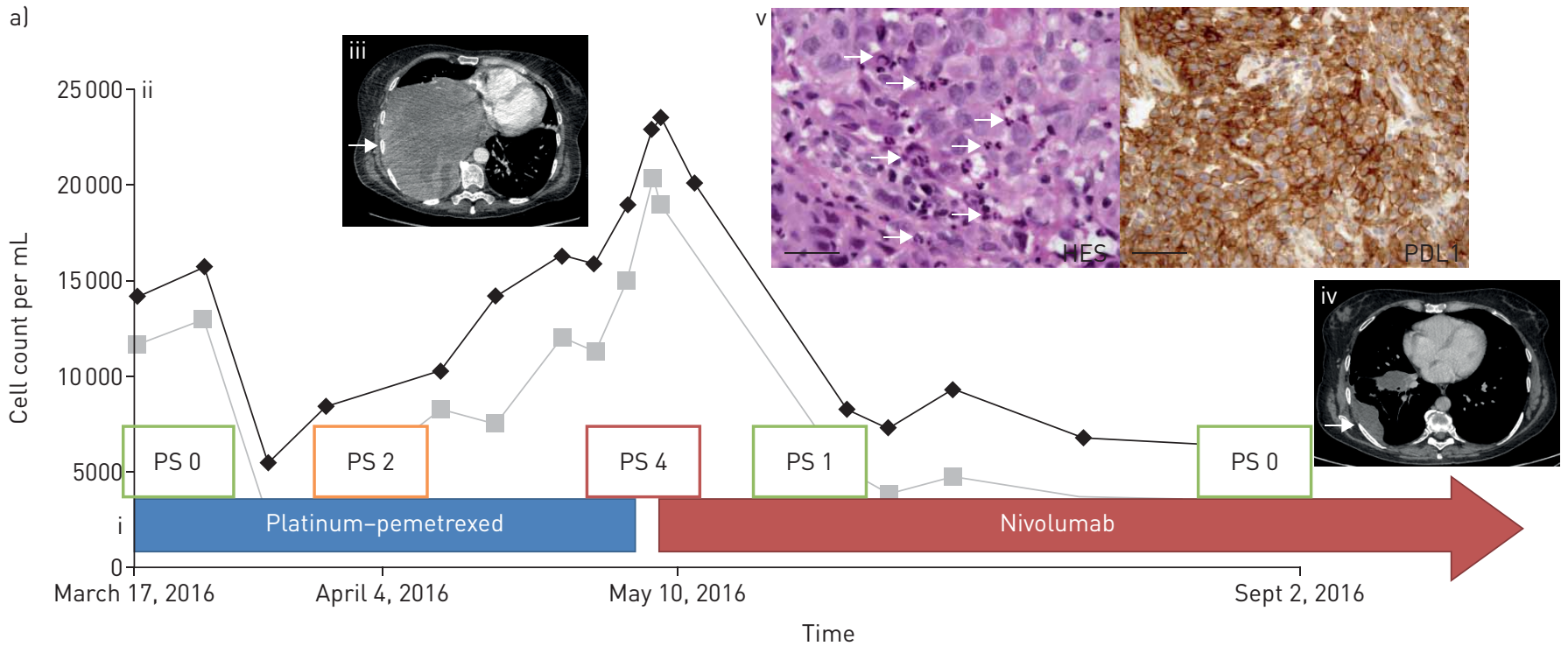

b)

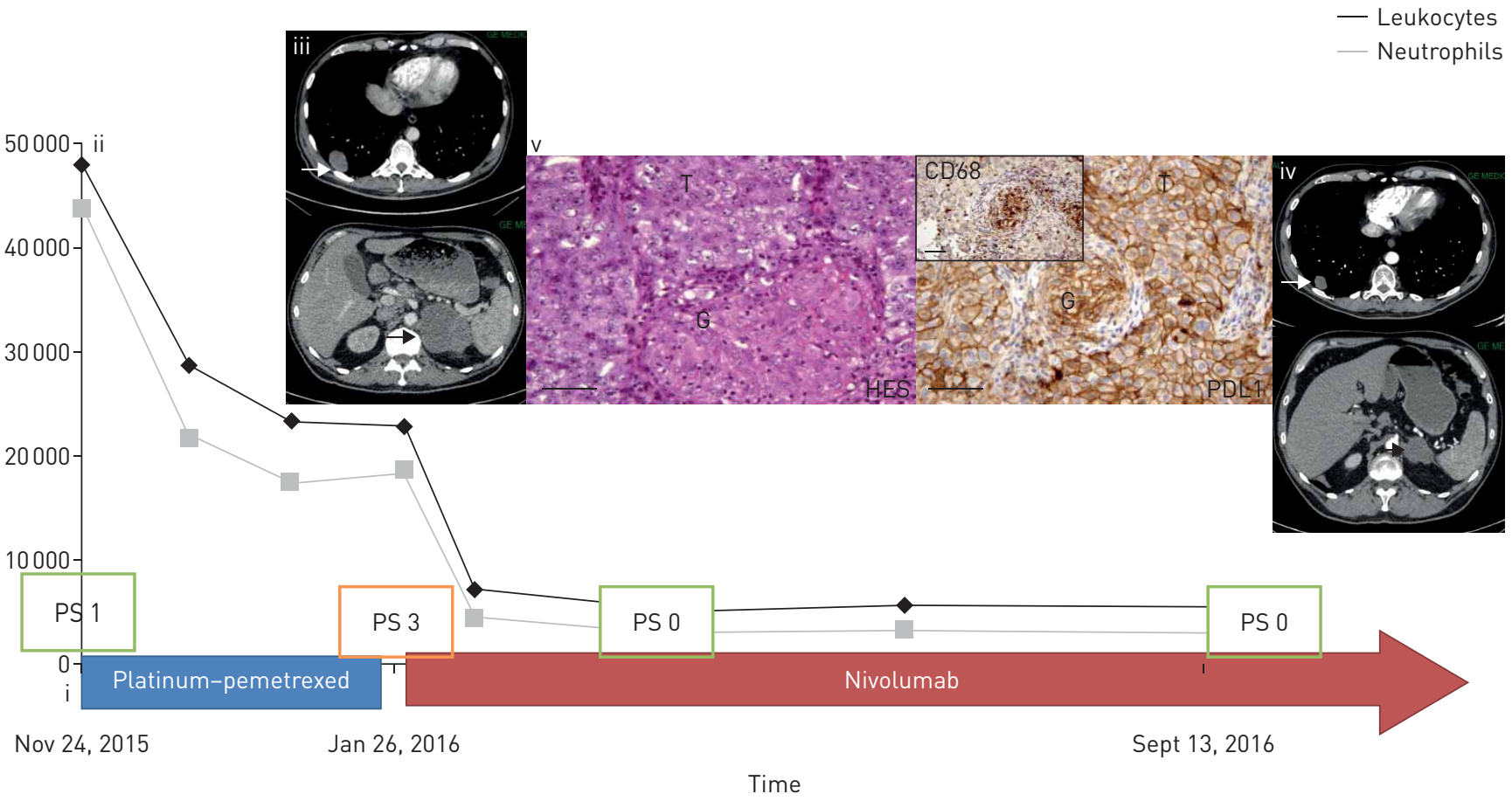

FIGURE 1 a) "Lazarus"-type response in a 47-year-old woman with metastatic nonsmall cell lung cancer (NSCLC): biological, pathological, clinical and radiologic findings timeline. i) Timeline of performance status (PS) evolution and therapies. ii) Leukocyte and neutrophil counts over time. iii) Computed tomography (CT) scan before starting nivolumab showing extensive mass in the right lung. iv) Latest findings of CT scan, with important partial response. v) Haematoxylin and eosin stain (HES) (left panels; scale bar=50 $\mu \mathrm{m}$ ) and tumour programmed death ligand 1 (PD-L1) immunostaining (right panels; scale bar $=50 \mu \mathrm{m}$ ), with rabbit mAb E1L3N monoclonal antibody from Cell Signaling Technology at 1/400 dilution using Leica BOND-MAX autostainer. Arrowheads indicate neutrophils infiltrating tumour cells. b) Rapid response for a man PS 3 with metastatic lung adenocarcinoma: biological, pathological, clinical and radiologic findings timeline. i) Timeline of PS evolution and therapies. ii) Leukocyte and neutrophil count over time. iii) CT scan before initiation of nivolumab, showing inferior right lobe mass, left adrenal gland metastasis. iv) Latest CT scan, showing persistent partial response. v) Positive HES (left panels; scale bar $=100 \mu \mathrm{m}$ ), and tumour PD-L1 immunostaining (right panels; scale bar $=100 \mu \mathrm{m}$ ) with rabbit mAb E1L3N monoclonal antibody from Cell Signaling Technology at 1/400 dilution using Leica BOND-MAX platform. Inset in upper right panel displays CD68 immunostaining (scale bar=50 $\mu \mathrm{m}$ ) (clone KP1 from Dako at 1/100 dilution), showing the granulomatous tumour stroma. T: tumour cells; G: granuloma.

alveolitis was established. The decision to initiate a short-term course of oral steroids was then taken, while nivolumab was re-initiated after a 30-day interruption, without pneumonitis relapse.

Patient 2 was a 52-year-old man, who was a heavy former smoker, and presented with abdominal pain and major leukocytosis (44000 per $\mathrm{mL}$ with $90 \%$ neutrophils) (figure 1b). A right inferior pulmonary 
lesion was found, associated with supraclavicular lymph node enlargement and left adrenal gland metastasis (cT2aN3M1b, UICC v.8). TTF-1-positive adenocarcinoma with an abundant granulomatous stroma was diagnosed by supraclavicular lymph node biopsy (figure $1 \mathrm{~b}$ iv). Molecular genotyping revealed a KRAS codon 12 activating mutation.

Prior to treatment initiation, the patient's ECOG PS score was 1. He received three cycles of carboplatin plus pemetrexed from November 2015 onwards, without experiencing any haematological toxicity except for Grade 2 anaemia. CT scan follow-up revealed disease progression, and his PS worsened to 3, which was accompanied by a systemic inflammatory syndrome revealed by persistent leukocytosis and a 10-fold increase in CRP levels compared with normal levels. Complementary pathological analysis demonstrated that $90 \%$ of the tumour cells in the supraclavicular lymph node biopsy performed prior to therapy expressed PD-L1 antigen at the cell plasma membrane, and an E1L3N immunohistochemistry assay also revealed PD-L1-positive staining of inflammatory stromal cells. Treatment was then switched to nivolumab. Around 15 days following the first nivolumab administration, the patient experienced a dramatic clinical and biological improvement: PS returned to 0 and leukocyte count normalised. At the time of writing, 14 months after nivolumab initiation, the patient had returned to work while still receiving nivolumab infusions every other week, with marked radiological response. No treatment-related side-effects were observed during the first 14 months of treatment, with extensive clinical, radiological and biological follow-up.

We report here on two patients with lung cancer presenting with systemic inflammatory response and poor general condition, who derived major and prolonged benefits from PD-1 blockade. For both patients, intense PD-L1 expression was revealed by immunohistochemistry of pre-therapeutic biopsies. During this same 12-month period, our multidisciplinary cancer board registry included 280 new patients with lung cancer diagnosed in our thoracic oncology department, of whom 175 had Stage IV NSCLC. Among these, there were only seven with PD-L1 immunostaining exceeding $80 \%$ and with PS 3-4, who were all administered nivolumab as salvage therapy. In addition to the first two cases described above, two further patients among these seven experienced marked tumour shrinkage and remarkable clinical improvement, whereas the three others died within 8 weeks.

As of yet, there is a lack of robust and reliable predictive markers enabling us to identify those patients most likely to benefit from PD-1/PD-L1 blockade. Both granulocyte colony-stimulating factor and granulocytemacrophage colony-stimulating factor were shown to be secreted by lung cancer cells in patients with paraneoplastic leukocytosis, potentially contributing to the activation of dendritic antigen-presenting cells $[4,5]$. Of note is that increased eosinophil levels at baseline were shown to be associated with positive response in patients with advanced-stage melanoma undergoing ipilimumab therapy [6].

Our patients had PS $0-1$ at diagnosis, and could have qualified nowadays for pembrolizumab first-line therapy, according to the Keynote-024 trial results [7], which were not yet published at the time. However, the general condition of both patients rapidly worsened with chemotherapy, and markedly altered PS is generally thought to be associated with worse immune response. Nivolumab was used as a rescue therapy, in contrast to most clinical trials, which recruit only patients with PS $0-1$. To the best of our knowledge, there has been no case report published so far describing a Lazarus-type response to anti-PD1 in poor-condition patients with NSCLC. Moreover, deterioration in general condition is widely considered to reflect an impaired cell-mediated immune response. However, the only data available to date are derived from the CA209-153 trial [8]. That trial recruited only patients with PS 0-2, and response rates and disease control (DC) did not differ depending on PS: partial response was achieved in $11 \%$ of patients with PS $0-1$ and $20 \%$ of patients with PS 2, while DC was noted in $44 \%$ and $46 \%$, respectively. Interestingly, patients with poor PS may thus develop an efficient immune response under PD-1 inhibitor treatment, resulting in improved quality of life and extended survival, provided that their tumours express high PD-L1 antigen levels. It should be emphasised that we did not observe any Grade 3-4 toxicities in our patients during a mean follow-up of 11 months, despite the patients' poor general condition. We therefore believe that future trials should be implemented to further assess the efficiency of immunotherapy in selected patients with poor general condition but high tumour PD-L1 expression.

Johan Pluvy ${ }^{1,2}$, Solenn Brosseau ${ }^{1,2}$, Charles Naltet ${ }^{1,2}$, Marie-Agnès Opsomer $^{3}$, Aurélie Cazes ${ }^{4}$, Claire Danel ${ }^{4}$, Antoine Khalil ${ }^{5}$, Gérard Zalcman ${ }^{1,2}$ and Valerie Gounant ${ }^{1,2}$

${ }^{1}$ Thoracic Oncology Dept, Paris-Diderot University, Bichat Hospital (APHP), Paris, France. ${ }^{2}$ Early Phase Trials Unit CIC14-25, CLIP2 Paris-Nord, Bichat Hospital (APHP), Paris, France. ${ }^{3}$ Pharmacy Dept, Bichat Hospital (APHP), Paris, France. ${ }^{4}$ Pathology Dept, Paris-Diderot University, Bichat Hospital (APHP), Paris, France. ${ }^{5}$ Radiology Dept, ParisDiderot University, Bichat Hospital (APHP), Paris, France.

Correspondence: Valérie Gounant, Service d'oncologie thoracique, Hôpital Bichat-Claude Bernard, 46 rue Henri Huchard, F-75018 Paris, France. E-mail: valerie.gounant@aphp.fr

Received: Dec 182016 | Accepted after revision: April 272017 
Conflict of interest: Disclosures can be found alongside this document at erj.ersjournals.com

\section{References}

1 Borghaei H, Paz-Ares L, Horn L, et al. Nivolumab versus docetaxel in advanced nonsquamous non-small-cell lung cancer. N Engl J Med 2015; 373: 1627-1639.

2 Brahmer J, Reckamp KL, Baas P, et al. Nivolumab versus docetaxel in advanced squamous-cell non-small-cell lung cancer. N Engl J Med 2015; 373: 123-135.

3 Rizvi NA, Mazieres J, Planchard D, et al. Activity and safety of nivolumab, an anti-PD-1 immune checkpoint inhibitor, for patients with advanced, refractory squamous non-small-cell lung cancer (CheckMate 063): a phase 2, single-arm trial. Lancet Oncol 2015; 16: 257-265.

4 Matsuguchi T, Okamura S, Kawasaki C, et al. Constitutive production of granulocyte colony-stimulating factor and interleukin- 6 by a human lung cancer cell line, KSNY: gene amplification and increased mRNA stability. Eur J Haematol 1991; 47: 128-133.

5 Sawyers CL, Golde DW, Quan S, et al. Production of granulocyte-macrophage colony-stimulating factor in two patients with lung cancer, leukocytosis, and eosinophilia. Cancer 1992; 69: 1342-1346.

6 Martens A, Wistuba-Hamprecht K, Geukes Foppen M, et al. Baseline peripheral blood biomarkers associated with clinical outcome of advanced melanoma patients treated with ipilimumab. Clin Cancer Res 2016; 22: 2908-2918.

7 Reck M, Rodríguez-Abreu D, Robinson AG, et al. Pembrolizumab versus chemotherapy for PD-L1-positive non-small-cell lung cancer. N Engl J Med 2016; 375: 1823-1833.

8 Waterhouse D, Horn L, Reynolds C, et al. P2.33: safety profile of nivolumab administered as 30-minute (min) infusion: analysis of data from Checkmate 153. J Thorac Oncol 2016; 11: S236. 